\title{
Response of a Fibre Optic Long Period Grating Operating at the Phase Matching Turning Point to the Deposition of a Nanostructured Coating
}

\author{
S.W. James, S.M. Topliss, S.C. Cheung and R.P. Tatam \\ Engineering Photonics Group, School of Engineering, Cranfield University \\ Cranfield, MK43 0AL, UK.
}

\begin{abstract}
The response of the transmission spectrum of a fibre optic long period grating operating near the phase matching turning point to the deposition of a nanostructured coating is investigated. The dual-resonant bands characteristic of operation in this regime are observed to show a high sensitivity to the thickness of the coating, but with opposite signs. Appropriate design of the device, based on the grating period, the refractive index and thickness of the coating and the fibre composition, can allow the sensitivity of the device to the optical thickness of the coating to be optimised.
\end{abstract}

\section{Keywords}

Fibre optic long period grating

\section{INTRODUCTION}

In recent years, the properties of fibre optic long period gratings (LPGs) with coatings of refractive index higher than the fibre cladding and of thickness of order $100 \mathrm{~nm}$ have been investigated extensively. For constant coating refractive index, the LPG's response to increasing coating thickness is characterised by a "mode transition region", in which the resonance bands in the LPG's transmission spectrum show high sensitivity to changes in coating thickness ${ }^{1}$. Similar effect are observed for changes in the refractive index of a coating of fixed thickness, facilitating the development of sensors based upon changes in the optical characteristics of the coating in response to a stimulus. LPGs also exhibit sensivities to measurands such as temperature ${ }^{2}, \operatorname{strain}^{2}$ and curvature ${ }^{3}$. It has been shown that, for highest sensitivity to such parameters, the LPG should be designed to couple light from the propagating core mode to a cladding mode at a wavelength near the phase matching turning point ${ }^{4}$, which is characterised by the generation of dual-resonant bands that show sensitivities of opposite sign.

In this paper we investigate the properties of LPGs operating at the phase matching turning point to the deposition of a nanostructured coating, and show that, by appropriate choice of LPG parameters and optical thickness of the coating, it is possible to ensure that the phase matching turning point and the mode transition region coincide, offering optimum sensitivity to change in the optical properties of the coating.

\section{LONG PERIOD GRATINGS}

An LPG is a core-cladding mode coupling device formed by a periodic modulation of the refractive index of the core of the optical fibre. The period of an LPG typically lies in the range $100 \mu \mathrm{m}-1 \mathrm{~mm}$, and acts to couple light from the core mode to a discrete set of symmetric cladding modes at wavelengths governed by the phase matching condition:

$$
\lambda_{(x)}=\left(n_{\text {core }}-n_{\text {clad }(x)}\right) \Lambda
$$

where $\lambda(x)$ represents the wavelength at which coupling occurs to the $L P_{0 x}$ mode, $n_{\text {core }}$ is the effective refractive index of the mode propagating in the core of the fibre, $\mathrm{n}_{\text {clad }(\mathrm{x})}$ is the effective index of the $\mathrm{LP}_{0 \mathrm{x}}$ cladding mode, and $\Lambda$ is the period of the grating. The cladding modes suffer high attenuation, with the result that the transmission spectrum of an LPG contains a series of resonant loss bands. The dependence of the phase matching condition upon the effective refractive index of the cladding mode results in the central wavelengths of the resonance bands exhibiting sensitivity to the refractive index of the surrounding material. 
The deposition of a nanostructured coating onto an LPG is characterised by a thickness for which the central wavelength of the resonance bands become highly sensitive to the coating's properties ${ }^{5,6}$. The coating itself can act as a waveguide, and the sensitivity of the LPG's transmission spectrum to the coating's properties is maximized when the optical thickness of the coating is such that a mode of the coating waveguide is phase matched to one of the lower order cladding modes. This thickness is typically of order $100 \mathrm{~nm}$. In this "mode transition region", the cladding modes undergo a reorganization process and the effective refractive indices of the cladding modes exhibit their peak sensitivity to the refractive index and thickness of the coating, causing the observed changes in resonance wavelengths ${ }^{5,6,9}$.

For coupling to higher order cladding modes, the phase matching condition of equation (1) has a turning point, such that it is possible to couple to the same cladding mode at two different wavelengths ${ }^{7}$. Calculation of the core and cladding modes' dispersions allows the prediction of the coupling wavelength as a function of the period of the grating using equation 1. The result of this analysis is plotted in figure 1 (a) for periods in the range $350 \mu \mathrm{m}-450 \mu \mathrm{m}$ and over a wavelength range $750 \mathrm{~nm}-1150 \mathrm{~nm}$. The data plotted in figure 1 were calculated using the LP approximation to calculate the dispersion of the core and cladding modes.

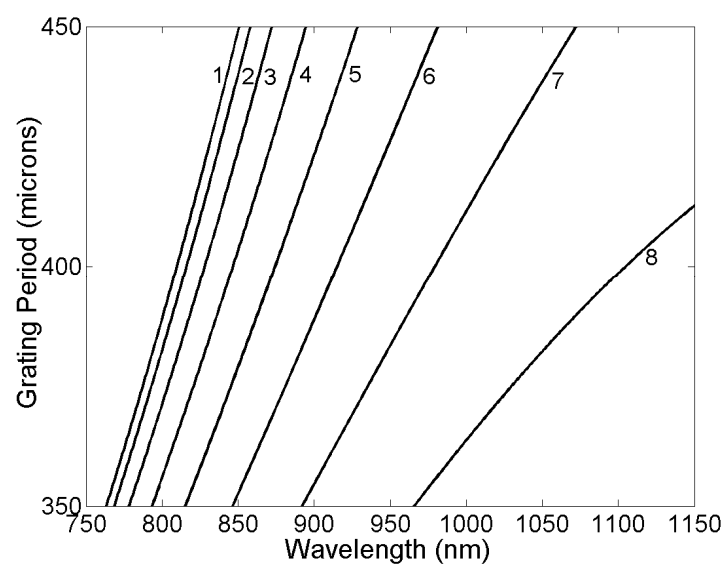

(a)

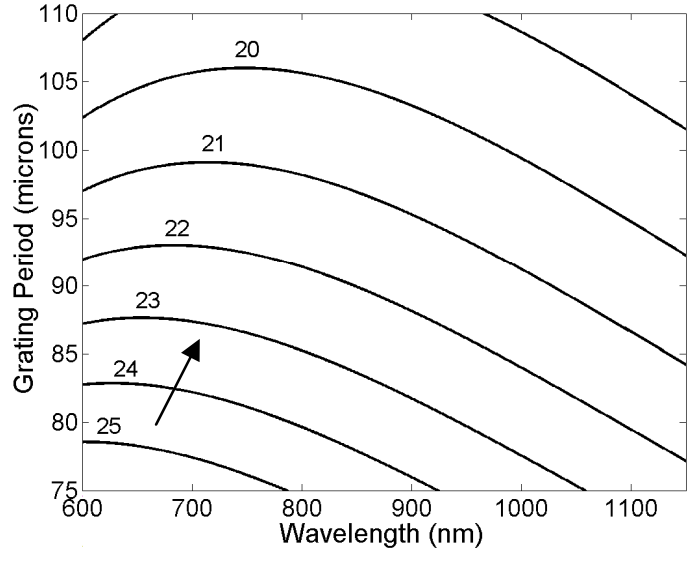

(b)

Figure 1. The relationship between the grating period and the wavelength at which coupling occurs to a set of symmetric cladding modes, assuming that the LPG was fabricated in an optical fibre of cut-off wavelength $670 \mathrm{~nm}$. (a) for the $1^{\text {st }} 8$ modes (b) for modes $20-25$. The numbers refer to the order of the cladding mode, $\mathrm{LP}_{0 \mathrm{x}}$.

In the range of periods depicted in figure 1 (a), the coupling is to the lower order cladding modes $\mathrm{LP}_{01}-\mathrm{LP}_{08}$, with the graphs showing a positive gradient across the wavelength range. For shorter periods, as shown in figure 1(b), coupling takes place to higher order cladding modes. The behaviour in this regime is different, with the phase matching condition showing a turning point in the wavelength range of interest. The wavelength at which the turning point occurs decreases with increasing period. It should be noted that all cladding modes will exhibit a turning point, however for the lower order modes this occurs at longer wavelengths, outside the range of interest here. An increase in the optical thickness of the coating causes the phase matching curves to move in the direction indicated by the arrow in figure 1(b).

If the period of the LPG is such that the phase matching turning point is accessed, then, as the surrounding refractive index increases, a broad attenuation band develops, with a fixed central wavelength, which subsequently splits into two resonance bands that shift in opposite directions as the refractive index is increased further. As the period changes, or the difference in the core and cladding mode effective indices change in response, for example, to a change in temperature or in the surrounding refractive index, the central wavelengths of the dual resonance bands move in opposite directions. It has been demonstrated theoretically and experimentally that the LPG resonance bands may exhibit their highest sensitivity to external perturbations at their phase matching turning point ${ }^{4}$. Thus to design a device to operate at a specified wavelength with high sensitivity to external perturbation, a cladding mode and period that produce a turning point at that wavelength should be selected. 


\section{EXPERIMENT}

LPGs of period $80 \mu \mathrm{m}$ and $100 \mu \mathrm{m}$ were fabricated in a single mode fibre of cut-off wavelength $670 \mathrm{~nm}$ using UV radiation at $266 \mathrm{~nm}$ from a frequency quadrupled Nd:YAG laser, using the point by point technique. The transmission spectrum was monitored by coupling the output from a tungsten halogen light bulb into the fibre and coupling the output from the fibre to a CCD spectrometer of resolution $0.3 \mathrm{~nm} .3 \mathrm{~nm}$ thick monolayers of $\omega$ - tricosenoic acid were deposited sequentially using the Langmuir-Blodgett technique, using the conditions described in [8], to achieve a maximum film thickness of $\sim 1 \mu \mathrm{m}$.

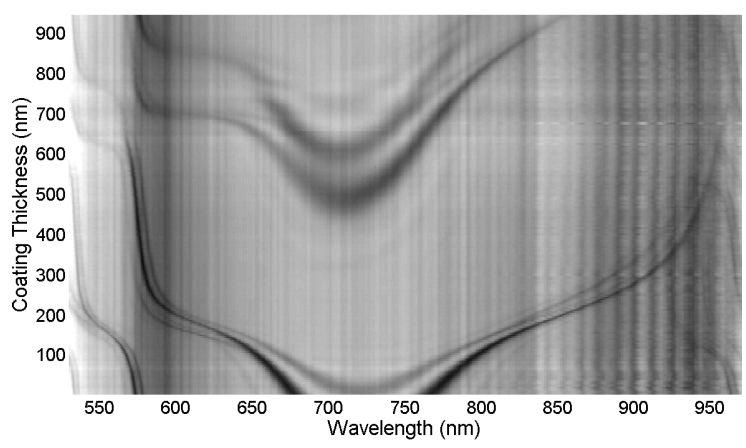

(a)

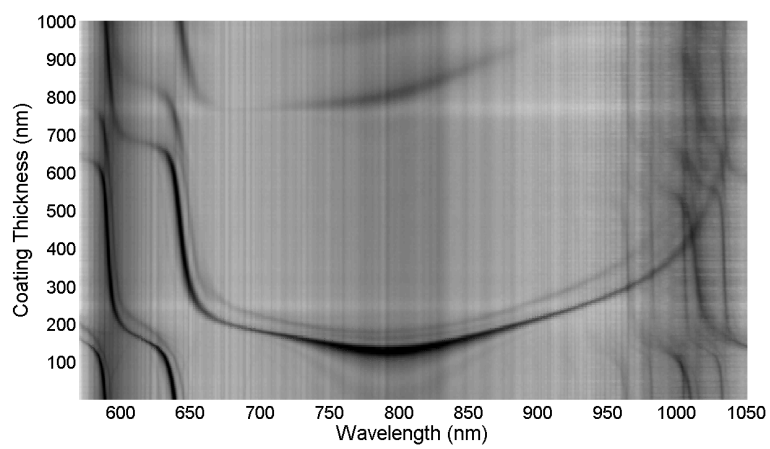

(b)

Figure 2 Evolution of the transmission spectrum of an LPG fabricated in a single mode optical fibre of cut-off wavelength $670 \mathrm{~nm}$, in response to the deposition of monolayers of $\omega$ - tricosenoic acid using the Langmuir-Blodgett technique (a) LPG period $80 \mu \mathrm{m}$, (b) LPG period $100 \mu \mathrm{m}$. The gray scale represents the measured transmission, with white corresponding to $100 \%$, and black to $0 \%$.

The form of the response of the central wavelengths of the resonance bands to layer by layer increases in the thickness of the coating deposited onto the LPG of period $80 \mu \mathrm{m}$ is illustrated in figure 2(a). The spectrum contains resonance bands corresponding to $1^{\text {st }}$ order coupling to the cladding modes. The resonance bands corresponding to coupling to the lower order cladding mode, originally centred at $575 \mathrm{~nm}$ in figure 3, exhibit the typical behaviour reported previously for nanoscale coating deposition onto $\mathrm{LPGs}^{8,9}$, in that the resonance bands undergo a blue shift, showing a region of high sensitivity that usually characterises the "mode transition region", in which one of the lower order cladding modes becomes phase matched to a mode of the waveguide formed by the coating material, and in which the effective indices of the cladding modes change rapidly ${ }^{5,6}$. For the $80 \mu \mathrm{m}$ period the phase matching condition is such that coupling to the $\mathrm{LP}_{024}$ cladding mode produces dual resonant bands in the absence of the coating, and the bands show opposite sensitivities to increasing coating thickness.

The response of the transmission spectrum of the LPG of period $100 \mu \mathrm{m}$ is shown in the grey scale images in figure 2(b). In this case, the dispersion turning point of mode $\mathrm{LP}_{024}$ is accessed at $800 \mathrm{~nm}$ for a coating thickness of order $200 \mathrm{~nm}$, which is coincident with the onset of the first mode-transition-region. Initially, there is no band in the $800 \mathrm{~nm}$ wavelength range. As the film thickness increases a broad attenuation band develops, with the extinction exhibiting a sensitivity to the coating thickness of $5 \% / \mathrm{nm}$, as plotted in figure 3. When this band is fully developed, further increases in coating thickness results in the attenuation band spliting into two, with the central wavelengths of the two bands shifting in opposite directions, as plotted in figure 4 . The peak sensitivity to coating thickness is $0.98 \mathrm{~nm} / \mathrm{nm}$ for the red shifted band, and $-1.45 \mathrm{~nm} / \mathrm{nm}$ for the blue shifted band, over a thickness range from $220 \mathrm{~nm}$ to $300 \mathrm{~nm}$. The measurement of the wavelength separation of the bands will allow further increased sensitivity. To illustrate the improvement in sensitivity, the highest sensitivity exhibited by a $1^{\text {st }}$ order coupling resonance band of a $400 \mu \mathrm{m}$ period $\mathrm{LPG}$, fabricated in the same type of fibre, being $0.5 \mathrm{~nm} / \mathrm{nm}^{9}$. 


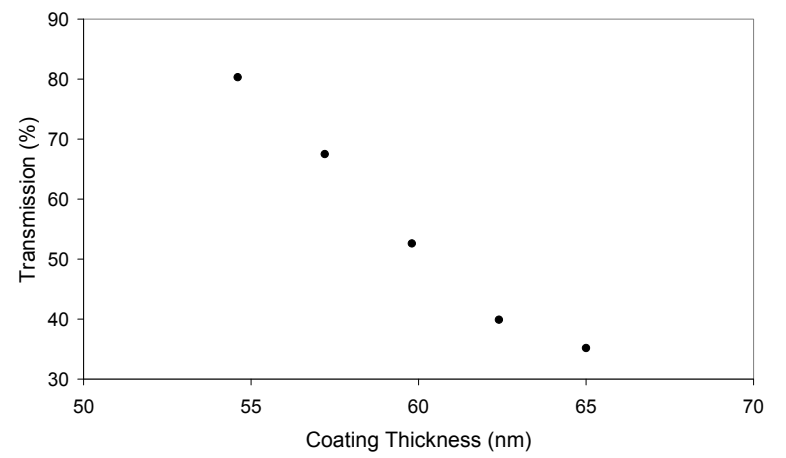

Figure 3: Transmission measured at the centre of the resonance band corresponding to coupling to the $\mathrm{LP}_{0,21}$ cladding mode as a function of the thickness of a coating of tricosenoic acid using the Langmuir Blodgett technique.

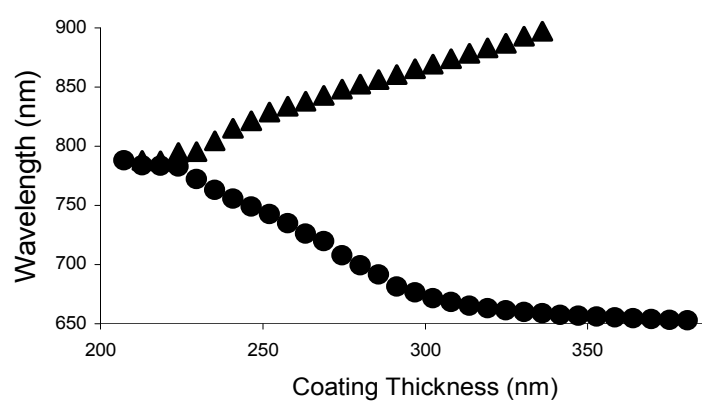

Figure 4: A plot of the wavelengths of the dual resonance bands as a function of the thickness of a coating of $\omega$ tricosenoic acid deposited using the Langmuir Blodgett technique onto an LPG of period $100 \mu \mathrm{m}$ fabricated in single mode optical fibre of cut off wavelength $700 \mathrm{~nm}$.

As the dual resonant bands show their highest sensitivity to external perturbation when the band is at the phase matching turning point, the coincidence of the generation of the dual resonance band with the onset of the $1^{\text {st }}$ transition region, where the cladding mode indices are most sensitive to the coating's optical properties, ensures that maximum sensitivity is obtained. Thus careful choice of the grating period, taking into account the fibre properties and the refractive index of the coating material is needed to ensure optimum sensitivity. While, in principle, the LPG sensor exhibits its highest sensitivity to all external perturbations at the phase matching turning point, it should be noted that, appropriate choice of core and cladding materials can allow the phase matching condition to specific cladding modes to be insensitive to, for example temperature, eliminating cross-sensitivity issues ${ }^{4}$. In addition, cross-sensitivity effects can be minimized by appropriate packaging or by exploiting the different sensitivities of each resonance band to different measurands ${ }^{2}$.

\section{SUMMARY}

An investigation of the response of LPG dual resonance bands near the dispersion turning point to the deposition of a nanostructured coating has been presented. Appropriate design of the device, taking into account the properties of the optical fibre, the period of the LPG and the refractive index of the coating material can ensure that mode dispersion turning point of the LPG coincides with the mode-transition-region that characterises LPGs with nanostructured coatings, ensuring optimum sensitivity to the coating's optical properties.

\section{REFERENCES}

1 S James and R Tatam Fibre Optic Sensors with Nano-Structured Coatings, J. Opt. A, 8 S340, 2006

$2 \mathrm{~V}$ Bhatia, Applications of long-period gratings to single and multi-parameter sensing, Opt. Express 4, 457-466, 1999

3 H Patrick, C Chang and S Vohra, Long period fibre gratings for structural bend sensing Electron. Lett. 18 1773-4, 1998

4 X Shu, L Zhang, and I Bennion, Sensitivity Characteristics of Long-Period Fibre Gratings J.Lightwave Technol. 20, 255-266, 2002

5 I Del Villar, I Matias and F Arregui, Influence on cladding mode distribution of overlay deposition on long period fibre gratings J. Opt. Soc. Am A 23, 651-658, 2006

6 A Cusano, A Iadicicco, P Pilla, L. Contessa, S. Campopiano, A. Cutolo, and M. Giordano, Mode transition in high refractive index coated long period gratings, Opt. Express 14, 19-34, 2006

7 X Shu, L Zhang and I Bennion, Sensitivity characteristics of long-period fiber gratings, J. Lightwave Technol. 20, 255-66, 2002.

$8 \mathrm{~N}$ Rees, S James, R Tatam, and G Ashwell Optical Fibre Long Period Gratings With Langmuir-Blodgett Thin Film Overlays Opt.Lett., 9, 686-688, 2002

$9 \mathrm{~S}$ James, C Cheung and R Tatam, Experimental observations on the response of 1st and 2nd order fibre optic long period grating coupling bands to the deposition of nanostructured coatings, Opt. Express, 15, 13096-13107, 2007 